\title{
Chlamydia pneumoniae disrupts lipid metabolism in human umbilical vein endothelial cells
}

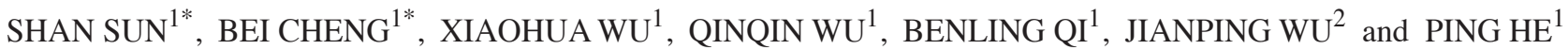 \\ Departments of ${ }^{1}$ Gerontology and ${ }^{2}$ Cardiology, Union Hospital, \\ Tongji Medical College of Huazhong University of Science and Technology, \\ Wuhan, Hubei 430022, P.R. China
}

Received September 5, 2013; Accepted May 9, 2014

DOI: $10.3892 / \mathrm{mmr} .2014 .2295$

\begin{abstract}
Atherosclerosis is well established as a chronic inflammatory disorder, and Chlamydia pneumoniae is considered to be a risk factor for atherosclerotic development. Endothelial dysfunction, caused by oxidized low-density lipoprotein (ox-LDL) is an early atherosclerotic marker. However, the effect of $C$. pneumoniae on lipid metabolism in vascular endothelial cells is yet to be elucidated. The aim of the present study was to investigate the effects of $C$. pneumoniae on lipid metabolism in human umbilical vein endothelial cells (HUVECs). In the present study, LDL oxidation was found to be significantly induced in the supernatant, but not the cell lysates, of $C$. pneumoniae-infected HUVECs. Furthermore, $C$. pneumoniae infection was observed to increase the levels of total cholesterol and cholesteryl esters in LDL-treated HUVECs. In addition, C. pneumoniae was found to upregulate the expression of scavenger receptor $\mathrm{A}$, cluster of differentiation 36 and acyl-coenzyme A: cholesterol acyltransferase $1 \mathrm{mRNA}$ and protein. C. pneumoniae was also observed to downregulate the mRNA and protein expression of ATP binding cassette transporter (ABC) A1 and ABCGl in LDL-treated HUVECs. These results show that $C$. pneumoniae disrupts lipid metabolism in HUVECs.
\end{abstract}

\section{Introduction}

Over recent decades, increasing evidence has suggested that infection may be an important risk factor for the development of atherosclerosis and coronary artery disease $(1,2)$. Epidemiological and clinical studies have demonstrated that infection with Chlamydia pneumoniae, an intracellular

Correspondence to: Professor Ping He, Department of Gerontology, Union Hospital, Tongji Medical College of Huazhong University of Science and Technology, 1277 Jie-Fang Avenue, Wuhan, Hubei 430022, P.R. China

E-mail: doctorpeace74@gmail.com

*Contributed equally

Key words: Chlamydia pneumoniae, endothelial cells, atherosclerosis, lipid metabolism organism, is an independent risk factor for cardiovascular diseases (3-5). Endothelial cells, vascular smooth muscle cells and monocytes/macrophages are involved in the atherogenic process, and infected peripheral blood monocytes can transmit the $C$. pneumoniae infection to smooth muscle and endothelial cells (6).

The endothelial layer provides a nonthrombogenic and nonadherent surface for circulating leukocytes, and is required for the maintenance of normal vessel function (7). Furthermore, vascular endothelial cells regulate complex processes, including hemostasis, fibrinolysis, inflammation, vessel tone, blood pressure, lipoprotein metabolism and angiogenesis (8). The accumulation of oxidized low-density lipoprotein (ox-LDL) in the arterial wall can activate the immune system and initiate a pro-inflammatory cascade of events, including the endogenous production of reactive oxygen species (ROS), endothelial dysfunction, the recruitment and trans-endothelial migration of monocytes with consequent transformation into foam cells and the proliferation of vascular smooth muscle cells. These events ultimately lead to atherosclerosis (9-11). Studies have shown that C. pneumoniae infection stimulates LDL oxidization and uptake and foam cell formation in macrophages $(12,13)$. Furthermore, a previous study by our group indicated that $C$. pneumoniae induced macrophage-derived foam cell formation through upregulation of the expression of scavenger receptor (SR) A1, cluster of differentiation (CD) 36 and acyl-coenzyme A: cholesterol acyltransferase 1 (ACAT1), and through downregulation of the expression of ATP binding cassette transporter (ABC) A1 and ABCGl (14). These genes regulate intracellular cholesterol homeostasis in vascular endothelial cells, including cholesterol synthesis, influx and efflux (15-17). However, few studies have investigated the effect of $C$. pneumoniae infection on endothelial lipid metabolism.

\section{Materials and methods}

Ethics statement. The present study involving fresh plasma was approved by normolipidemic volunteers and the Wuhan Blood Centre (Wuhan, China; authorization: 2010-8) and conformed to the guidelines outlined in the Declaration of Helsinki.

Reagents and antibodies. HUVECs and epithelial HEp-2 cells were obtained from Tongji Medical College (Wuhan, 
China). C. pneumoniae strain AR-39 was purchased from the American Type Culture Collection (Rockville, MA, USA). Dulbecco's Modified Eagle's Medium (DMEM) and fetal bovine serum (FBS) were purchased from Hyclone (Thermo Fisher Scientific, Inc., Waltham, MA, USA). A cell lipid peroxide assay kit was purchased from Genmed Scientifics, Inc. (Shanghai, China). A quantitative polymerase chain reaction (qPCR) kit was purchased from Takara Bio Inc. (Otsu, Shiga, Japan). Fluorescein isothiocyanate (FITC)-conjugated specific anti-chlamydial monoclonal antibodies were obtained from Dako Ltd. (Copenhagen, Denmark), and mouse polyclonal anti-SR-A1 antibodies were obtained from Santa Cruz Biotechnology, Inc. (Dallas, TX, USA). Rat monoclonal anti-CD36 antibodies were obtained from R\&D Systems, Inc. (Minneapolis, MN, USA). Rabbit polyclonal anti-ACAT1 antibodies were obtained from Cayman Chemical Co. (Ann Arbor, MI, USA). Rabbit monoclonal anti-ABCG1 antibodies were obtained from Epitomics (Burlingame, CA, USA). Mouse monoclonal anti-ABCA1 antibodies were obtained from Abcam PLC (Cambridge, UK). Mouse polyclonal anti- $\beta$-actin antibodies and horseradish peroxidase (HRP)-conjugated secondary antibodies were obtained from AntGene Biotech Co., Ltd. (Wuhan, China).

Propagation of $C$. pneumoniae. C. pneumoniae strain AR-39 was propagated in HEp-2 cells in the presence of $2 \mu \mathrm{g} / \mathrm{ml}$ cycloheximide using centrifugation-driven infection as described previously (18). Infected HEp-2 cells were harvested subsequent to incubation for $72 \mathrm{~h}$ at $37^{\circ} \mathrm{C}$ and $5 \% \mathrm{CO}_{2}$, and disrupted by freezing, thawing and ultrasonication. Following centrifugation at $500 \mathrm{x} \mathrm{g}$ at $4^{\circ} \mathrm{C}$ for $30 \mathrm{~min}$ to remove the cell debris, the supernatant containing elementary bodies was collected and suspended in sucrose-phosphate-glutamic acid buffer and stored at $-70^{\circ} \mathrm{C}$ in aliquots until use. Chlamydial inclusion forming units (IFU) were assessed by counting the chlamydial inclusions formed in the HEp-2 cells using FITC-conjugated anti-chlamydial antibodies.

Infection and culture of HUVECs. HUVECs were seeded onto six-well plates at a density of $1 \times 10^{6}$ cells per well in DMEM containing $10 \% \mathrm{FBS}$ at $37^{\circ} \mathrm{C}$ in $5 \% \mathrm{CO}_{2}$. In the presence of $50 \mathrm{mg} / \mathrm{l} \mathrm{LDL}$, HUVECs were cultured alone or with $C$. pneumoniae $\left(1 \times 10^{6} \mathrm{IFU}\right)$ for $48 \mathrm{~h}$. In certain experiments, HUVECs were incubated in six-well plates with $50 \mathrm{mg} / \mathrm{l}$ ox-LDL for $48 \mathrm{~h}$.

LDL isolation and ox $-L D L$ preparation. Human LDL (density, 1.006-1.063 g/ml) was isolated from the blood of healthy donors using density-gradient ultracentrifugation as described previously (19). The isolated LDL was dialyzed in phosphate-buffered saline for $24 \mathrm{~h}$ and was condensed using polyoxyl 20,000. The concentration of LDL was measured using the Bradford assay, and the LDL underwent sterile filtration $(0.22 \mathrm{~mm})$ prior to storage in the dark at $4{ }^{\circ} \mathrm{C}$ for no longer than 2 weeks before use. LDL was analyzed using 4-20\% SDS-PAGE gradient gel and Sudan black B staining to reveal a single protein. The preparations of ox-LDL from human plasma were performed as described previously (20). Briefly, LDL was diluted to a concentration of $200 \mathrm{mg} / \mathrm{dl}$ in medium M199 and incubated with $5 \mu \mathrm{mol} / 1 \mathrm{CuSO}_{4}, \mathrm{CuCl}_{2}$ or copper acetate at $37^{\circ} \mathrm{C}$ for $24 \mathrm{~h}$, as indicated. The reactions were terminated using the addition of $100 \mu \mathrm{mol} / 1$ EDTA. The oxidation state of LDL was measured using the PeroXOquant Quantitative Peroxide Assay kit (Pierce Biotechnology, Inc., Rockford, IL, USA). Peroxidation products contained in the LDL and ox-LDL were $<5$ and 200-300 $\mu \mathrm{mol} / 1 \mathrm{H}_{2} \mathrm{O}_{2}$, respectively. Contamination of LDL and the modified LDL preparations by the lipopolysaccharide (LPS) endotoxin was assessed using a Limulus Amebocyte Lysate kit (BioWhittaker ${ }^{\circledR}$; Lonza, Walkersville, MD, USA). All LDL preparations had an LPS content of $<50 \mathrm{pg} / \mathrm{mg}$ protein.

Measurement of thiobarbituric acid reactive substances (TBARS). The determination of LDL oxidation was performed as described previously (21). The determination of TBARS involves the measurement of malondialdehyde (MDA) derived from the hydroperoxidation of unsaturated fatty acids containing three or more double bonds (22). In brief, $2.5 \mathrm{ml} 10 \%$ trichloroacetic acid was added to a tube with $0.5 \mathrm{ml}$ HUVEC supernatant or cell lysates with or without $C$. pneumoniae infection, in order to precipitate the protein. Following centrifugation, $100 \mu 1$ supernatant was mixed with $50 \mu \mathrm{l} 0.67 \%$ TBA. Subsequent to vortexing and boiling for $15 \mathrm{~min}$, samples were cooled under running water. Absorbance was measured at $532 \mathrm{~nm}$ in a Perkin Elmer Lambda 2 spectrophotometer (Perkin Elmer, Überlingen, Germany) against a blank control with distilled water. The concentration of oxidation products was determined as MDA (nmol/ml) according to a standard curve of MDA reacting with TBA using the following formula: MDA $(\mathrm{nmol} / \mathrm{ml})=$ absorbance $x$ 53.4188. Experiments were repeated three times.

Intracellular cholesterol measurement. Cells were harvested and disrupted using ultrasonication. Intracellular TC and free cholesterol (FC) were detected using zymochemistry as described previously (23). TC and FC content was determined using a standard curve $(312.5,625,1,250$ and 2,500 $\mu \mathrm{mol} / 1$ standard cholesterol) and expressed as $\mu \mathrm{mol} / \mathrm{g}$ protein. CE content was calculated by subtracting the content of FC from that of TC. Experiments were repeated three times.

$q P C R$ analysis. Total RNA was isolated using TRIzol ${ }^{\circledR}$ reagent (Takara Bio, Inc.). cDNA was synthesized using a PrimeScript $^{\mathrm{TM}}$ RT Reagent kit (Takara Bio, Inc.) according to the manufacturer's instructions, and qPCR was performed using a Taq polymerase (Takara Bio, Inc.). The oligonucleotide primers for SR-A1, CD36, ACAT1, ABCA1, ABCG1 and $\beta$-actin were as follows: SR-A1, 5'-GCAGTTCTCATC CCTCTCAT-3' (forward) and 5'-GGTATTCTCTTGGAT TTTGCC-3' (reverse); CD36, 5'-TGCCTCTCCTAG TTGAAAAC-3' (forward) and 5'-GCAACAAACGATCAC CACAC-3' (reverse); ACAT1, 5'-TCCCAGGAATCCCAC TGTAA-3' (forward) and 5'-ACGAAGAGCACGGGA TAGAA-3' (reverse); ABCA1, 5'-TATGAGGGCCAGATC ACCTC-3' (forward) and 5'-GCTGGCTTGTTTTGCTT TTC-3' (reverse); ABCG1, 5'-CAGGAAGATTAGACACTG TGG-3' (forward) and 5'-GAAAGGGGAATGGAGAGAAG-3' (reverse); $\beta$-actin, 5'-GTCCACCTTCCAGCAGATGT-3' (forward) and 5'-CACCTTCACCGTTCCAGTTT-3' (reverse). 
Western blot analysis. Total protein was extracted from the cells and the protein content was measured in triplicate using the Bicinchoninic Acid Protein Assay kit (Pierce Biotechnology, Inc.). Absorption was measured at $562 \mathrm{~nm}$. Protein samples $(60 \mu \mathrm{g})$ were separated using $10 \%$ SDS-PAGE and transferred to polyvinylidene fluoride membranes (Invitrogen Life Technologies, Grand Island, NY, USA). Membranes were incubated at $4^{\circ} \mathrm{C}$ overnight with the following antibodies: Mouse polyclonal anti-SR-A1 (dilution, 1:200), rat monoclonal anti-CD36 (dilution, 1:500), rabbit polyclonal anti-ACAT1 (dilution, 1:200), mouse monoclonal anti-ABCA1 (dilution, 1:1,000), rabbit monoclonal anti-ABCG1 (dilution, $1: 2,000)$ and mouse polyclonal anti- $\beta$-actin (dilution, 1:1,000). The membranes were then incubated with HRP-conjugated secondary anti-rabbit, -rat and -mouse antibodies, each at a dilution of 1:2,000, at room temperature for $2 \mathrm{~h}$. Proteins were detected using the enhanced chemiluminescence method. For quantification, SR-A1, CD36, ACAT1, ABCA1 and ABCG1 protein levels were normalized to those of $\beta$-actin.

Statistical analysis. Quantitative data are expressed as the mean \pm standard derivation. Direct comparisons between two groups were performed using the Student's t-test. Data from more than two groups were compared using analysis of variance with repeated measures, followed by the Student-Newman-Keuls multiple comparison test. A value of $\mathrm{P}<0.05$ was considered to indicate a statistically significant difference.

\section{Results}

Increased lipid peroxidation products in the supernatants, but not cell lysates, of C. pneumoniae-infected HUVECs. Lipid peroxidation was quantified using the measurement of TBARS. As shown in Fig. 1A, lipid peroxidation products were increased in the supernatant of $C$. pneumoniae-infected HUVECs $\left(1 \times 10^{6}\right.$ IFU). MDA production was observed to increase from $4.61 \pm 0.90 \mathrm{nmol} / \mathrm{ml}$ in the control HUVEC supernatant to $7.16 \pm 0.40 \mathrm{nmol} / \mathrm{ml}$ in the infected HUVEC supernatant $(\mathrm{P}<0.05)$. In contrast to the supernatant, no significant difference in LDL oxidation was observed in the infected HUVEC lysates (Fig. 1B; P>0.05).

Effect of C. pneumoniae on intracellular cholesterol metabolism. Cholesterol is accumulated either as CE in cytoplasmic vesicles or as FC in the membrane (24). To determine whether intracellular cholesterol metabolism was affected by $C$. pneumoniae in HUVECs, the levels of intracellular TC and CE were assessed using lipid mass quantification. LDL-treated HUVECs were used as a negative control, ox-LDL-treated HUVECs were used as a positive control and LDL-treated HUVECs infected with C.pneumoniae were used as the infection group. As shown in Table I, C. pneumoniae infection was found to increase the levels of TC and CE compared with the negative control $(\mathrm{P}<0.05)$. In addition, levels of intracellular TC and CE were increased in the ox-LDL-treated HUVECs compared with those in the negative control group $(\mathrm{P}<0.05)$. C. pneumoniae infection was not observed to significantly increase TC and CE levels compared with the positive controls $(\mathrm{P}>0.05)$.
A

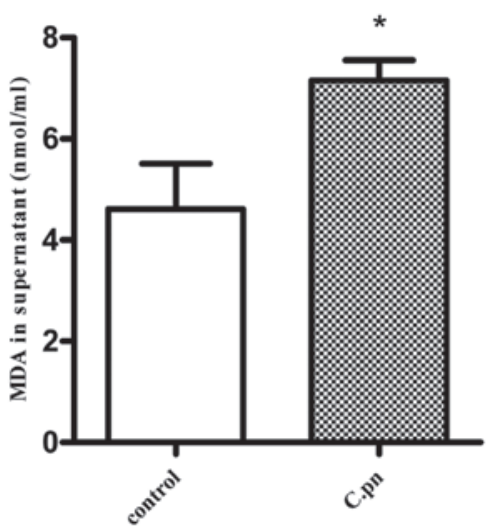

B

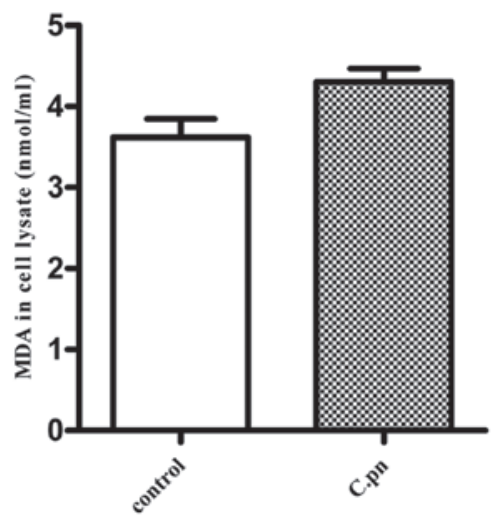

Figure 1. MDA production in the (A) supernatant and (B) cell lysates of control and C.pn-infected HUVECs. In the presence of $50 \mathrm{mg} / 1 \mathrm{LDL}, \mathrm{HUVECs}$ were cultured alone or with C.pn ( $1 \times 10^{6}$ inclusion forming units) for $48 \mathrm{~h}$. The LDL-treated HUVECs were used as controls. The increase in lipid peroxidation products was expressed as an increase in MDA production. Data are presented as the mean \pm standard deviation $(n=3)$. ${ }^{*} \mathrm{P}<0.05$ versus control HUVECs, human umbilical vein endothelial cells; MDA, malondialdehyde; LDL, low-density lipoprotein; C.pn, Chlamydia pneumoniae.

SR-A1 and CD36 expression is upregulated in LDL-treated HUVECs with $C$. pneumoniae infection. The mRNA and protein expression of lipoprotein receptors was assessed using qPCR and western blot analyses, respectively. SR-A1 and CD36 have been demonstrated to participate in lipoprotein uptake (25). As shown in Fig. 2A and B, SR-A1 and CD36 mRNA expression was upregulated by up to 1.8 - and 2.0 -fold in the $C$. pneumoniae-infected HUVECs compared with that in the negative controls $(\mathrm{P}<0.05)$. In addition, ox-LDL was found to upregulate the mRNA expression of SR-A1 and CD36 by up to 2.0 - and 1.5 -fold, respectively $(\mathrm{P}<0.05)$. However, no significant difference was observed in the mRNA expression of SR-A1 and CD36 between the $C$. pneumoniae-infected HUVECs and the positive controls $(\mathrm{P}>0.05)$. Furthermore, $C$. pneumoniae infection was found to increase the protein expression of SR-A1 and CD36 by 3.53- and 2.40-fold, respectively, relative to the negative control group $(\mathrm{P}<0.05)$. SR-A1 and CD36 protein expression was also increased by up to 4.12- and 2.11-fold, respectively, in the positive control group relative to that in the negative controls $(\mathrm{P}<0.05)$ (Fig. 2C and D). No significant difference was observed in the protein expression of SR-A1 and CD36 between the $C$. pneumoniae-infected HUVECs and the positive controls ( $\mathrm{P}>0.05)$. 
Table I. Effect of Chlamydia pneumoniae on TC and CE levels in human umbilical vein endothelial cells.

\begin{tabular}{lcccc}
\hline Groups & TC $(\mu \mathrm{mol} / \mathrm{g})$ & $\mathrm{FC}(\mu \mathrm{mol} / \mathrm{g})$ & $\mathrm{CE}(\mu \mathrm{mol} / \mathrm{g})$ & $\mathrm{CE} / \mathrm{TC}(\%)$ \\
\hline LDL & $35.7 \pm 0.8$ & $24.4 \pm 0.6$ & $11.3 \pm 1.1$ & 31.7 \\
LDL+C.pn & $74.5 \pm 3.1^{\mathrm{a}}$ & $41.3 \pm 1.7$ & $33.2 \pm 2.1^{\mathrm{a}}$ & 44.6 \\
ox-LDL & $76.5 \pm 2.6^{\mathrm{a}}$ & $39.5 \pm 1.2$ & $36.7 \pm 0.9^{\mathrm{a}}$ & 48.2 \\
\hline
\end{tabular}

Data are presented as the mean \pm standard deviation of three independent experiments. ${ }^{\mathrm{a}} \mathrm{P}<0.05$ versus control (LDL group). TC, total cholesterol; CE, cholesteryl ester; FC, free cholesterol; LDL, low-density lipoprotein; ox-LDL, oxidized LDL; C.pn, Chlamydia pneumoniae.

A

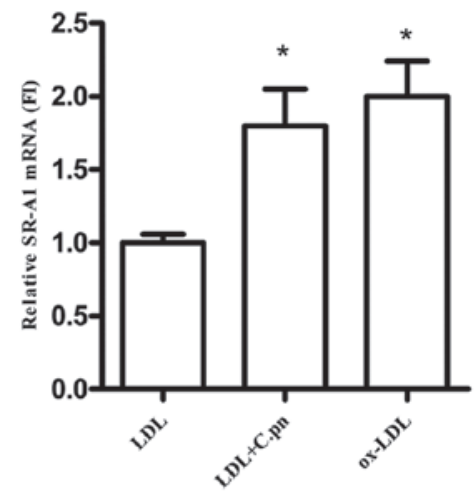

C
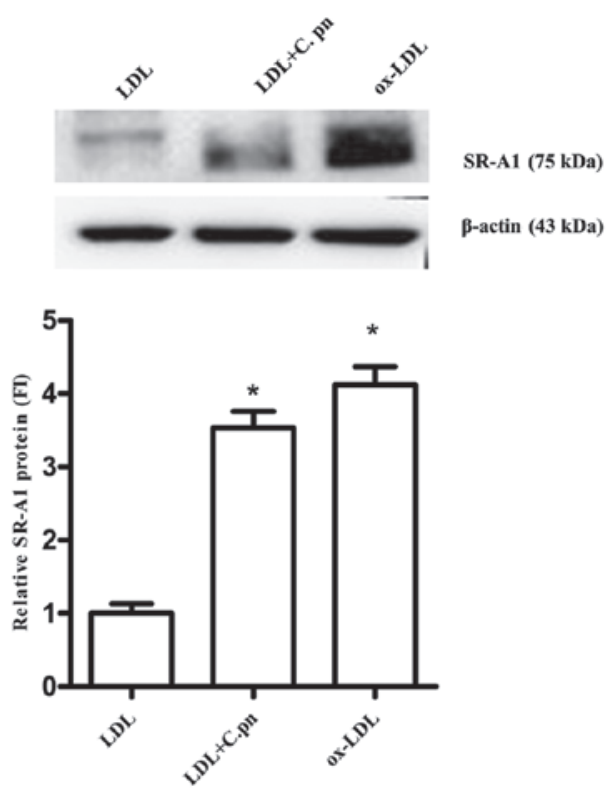

B

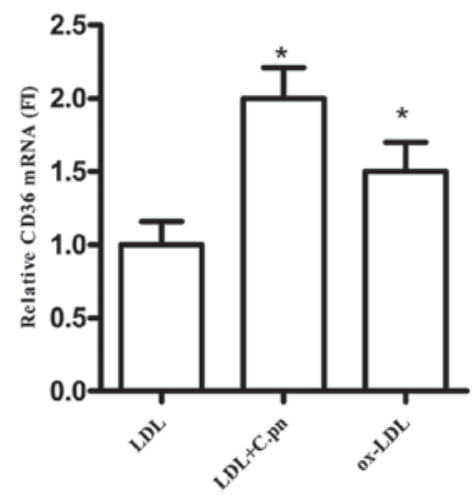

D
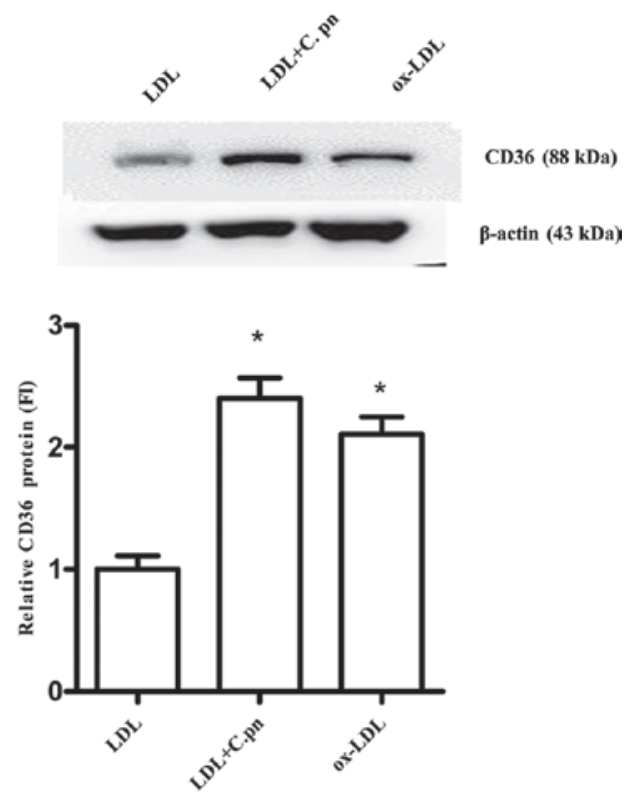

Figure 2. C.pn upregulates SR-A1 and CD36 expression in LDL-treated HUVECs. In the presence of $50 \mathrm{mg} / 1 \mathrm{LDL}$ or ox-LDL, HUVECs were cultured alone or with C.pn (1x106 inclusion forming units) for $48 \mathrm{~h}$. (A and B) Quantitative polymerase chain reaction analysis of (A) SR-A1 and (B) CD36 mRNA expression relative to that of $\beta$-actin. (C and D) Western blot analysis of (C) SR-A1 and (D) CD36 protein expression relative to that of $\beta$-actin. Data are expressed as the FI of the control (control=1 arbitrary unit). Data are presented as the mean \pm standard deviation from three independent experiments. "P<0.05 versus control (LDL group). HUVECs, human umbilical vein endothelial cells; LDL, low-density lipoprotein; ox-LDL, oxidized LDL; C.pn, Chlamydia pneumoniae; SR, scavenger receptor; $\mathrm{CD}$, cluster of differentiation; FI, fold induction.

ACAT1 expression is upregulated in LDL-treated HUVECs with $C$. pneumoniae infection. The conversion of cholesterol to $\mathrm{CE}$ is catalyzed by the enzyme ACAT. In order to determine the role of ACAT1 in the $C$. pneumoniae-induced perturbation of intracellular cholesterol homeostasis in HUVECs, the expression of ACAT1 was assessed using qPCR and western blot analyses. As shown in Fig. $3 \mathrm{~A}$ and $\mathrm{B}, \mathrm{C}$. pneumoniae infection $\left(1 \times 10^{6}\right.$ IFU) was found to significantly increase the
mRNA and protein expression of ACAT1 by up to 1.5- and 1.9-fold, respectively, compared with the LDL-treated HUVECs $(\mathrm{P}<0.05)$. In addition, ox-LDL upregulated ACAT1 mRNA expression by up to 1.58 -fold and ACAT1 protein expression by up to 2.03-fold relative to the negative control. No significant difference was observed in ACAT1 mRNA and protein expression between the $C$. pneumoniae-infected HUVECs and the positive controls $(\mathrm{P}>0.05)$. These data show 
A

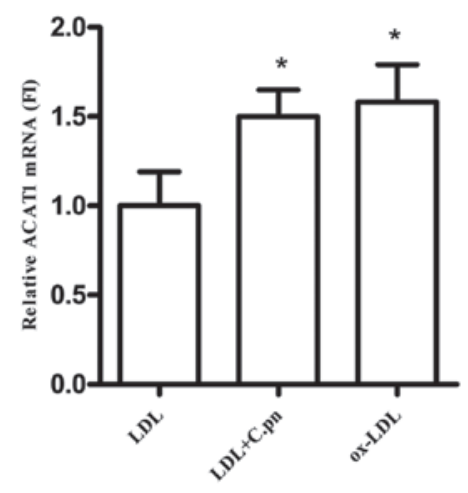

B
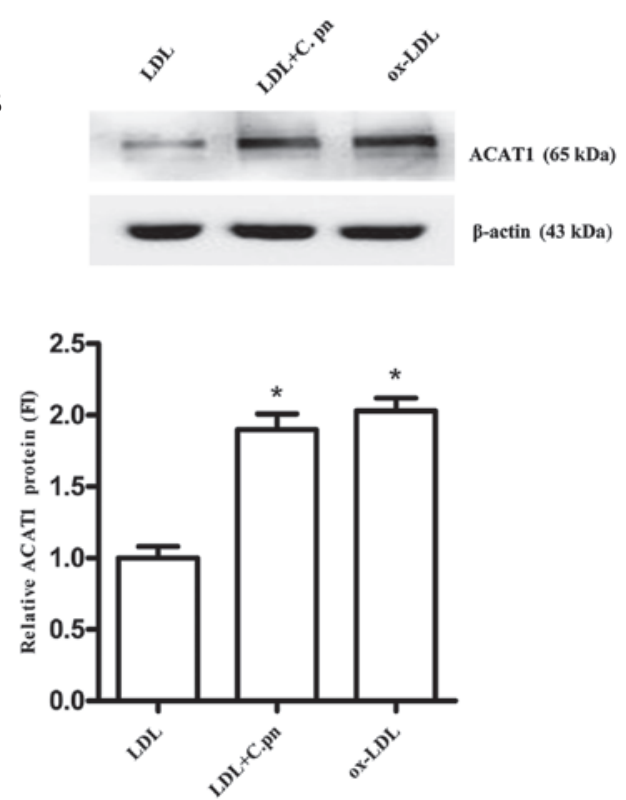

Figure 3. C.pn upregulates ACAT1 expression in LDL-treated HUVECs. In the presence of $50 \mathrm{mg} / 1 \mathrm{LDL}$ or ox-LDL, HUVECs were cultured alone or with C.pn (1x10 inclusion forming units) for $48 \mathrm{~h}$. (A) Quantitative polymerase chain reaction analysis of ACAT1 mRNA expression relative to that of $\beta$-actin. (B) Western blot analysis of ACAT1 protein expression relative to that of $\beta$-actin. Data are expressed as the FI of the control (control=1 arbitrary unit). Data are presented as the mean \pm standard deviation from three independent experiments. "P $<0.05$ versus control (LDL group). HUVECs, human umbilical vein endothelial cells; LDL, low-density lipoprotein; ox-LDL, oxidized LDL; C.pn, Chlamydia pneumoniae; ACAT1, acyl-coenzyme A: cholesterol acyltransferase 1; $\mathrm{FI}$, fold induction.

that ACAT1 may be involved in the $C$. pneumoniae-induced increases in CE levels in HUVECs.

ABCAl and ABCGl expression is downregulated in LDL-treated HUVECs with C. pneumoniae infection. ABCA1 and ABCG1 are critical cell surface proteins that mediate endothelial cholesterol efflux (15). In order to investigate the expression and regulation of ABCA1 and ABCG1 in C. pneumoniae-infected HUVECs, the mRNA and protein levels of ABCA1 and ABCG1 were detected. As shown in Fig. 4A and B, the mRNA expression of both ABCA1 and ABCG1 was reduced by $40 \%$ in the $C$. pneumoniae-infected HUVECs compared with that in the LDL-treated HUVECs $(\mathrm{P}<0.05)$. Furthermore, in the ox-LDL-treated HUVECs, the mRNA expression of ABCA1 and ABCG1 was reduced by $50 \%$ $(\mathrm{P}<0.05)$. In addition, ABCA1 and ABCG1 protein expression was reduced by 62 and $48 \%$ in the $C$. pneumoniae-infected HUVECs ( $\mathrm{P}<0.05$; Fig. 4C and D). However, no significant difference was observed between the $C$. pneumoniae-infected group and the positive control group $(\mathrm{P}>0.05)$.

\section{Discussion}

Atherosclerosis is an inflammatory disease in its genesis and progression, and it is initiated by the retention of lipids. The present study revealed that the supernatant of C. pneumoniae-infected HUVECs exhibited significant LDL oxidation, and that $C$. pneumoniae increased the intracellular levels of TC and CE through the regulation of target genes affecting lipid metabolism in endothelial cells. The rate of lipid peroxidation was determined using the TBARS method, which revealed that the supernatant, but not the cell lysate, of $C$. pneumoniae-infected HUVECs exhibited significant
LDL oxidation. Furthermore, $C$. pneumoniae infection was found to significantly increase the levels of TC and CE in the LDL-treated HUVECs; this finding was similar to the results for the ox-LDL-treated HUVECs. The present study also demonstrated that in vitro infection with $C$. pneumoniae in LDL-treated HUVECs significantly increased the expression of SR-A1, CD36 and ACAT1, which participate in intracellular lipid influx and CE synthesis. In addition, C.pneumoniae was observed to decrease the expression of ABCA1/G1, which mediate intracellular lipid efflux. Overall, the present study has indicated that $C$. pneumoniae disrupts lipid metabolism in HUVECs.

The importance of ox-LDL in terms of atherogenesis is well established. ox-LDL inhibits the expression of the constitutive endothelial nitric oxide synthetase enzyme and leads to oxidative stress-induced vascular endothelial cell apoptosis $(26,27)$. The release of ROS induces the expression of adhesion molecules on endothelial cells (28). Several studies have reported that infected monocytes/macrophages are capable of transmitting $C$. pneumoniae infection to endothelial cells and that $C$. pneumoniae infection of macrophages enhances their adhesion to the endothelium $(29,30)$. In addition, ox-LDL has been reported to enhance the expression of vascular adhesion molecules in $C$. pneumoniae-infected endothelial cells (31); the increased expression of vascular adhesion molecules consequently promotes the attraction and adherence of monocytes and T lymphocytes to the endothelium, which is a key process in the progression of arteriosclerosis (32). In the present study, the supernatant of $C$. pneumoniae-infected HUVECs was found to exhibit significant LDL oxidation. Thus, $C$. pneumoniae may induce LDL oxidation and initiate endothelial dysfunction chronically and continuously, eventually leading to atherogenesis. 
A

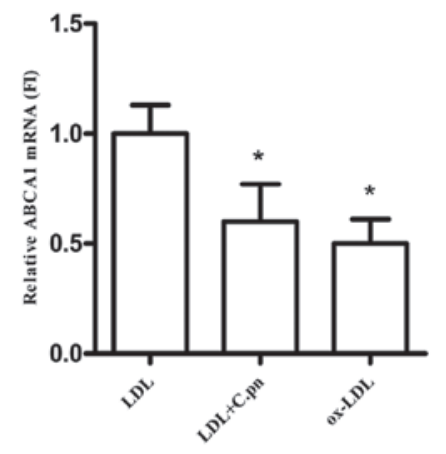

C
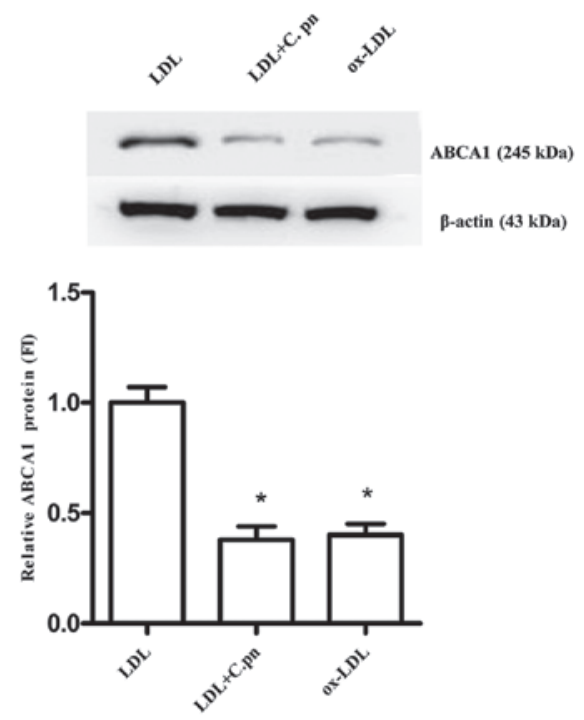

B
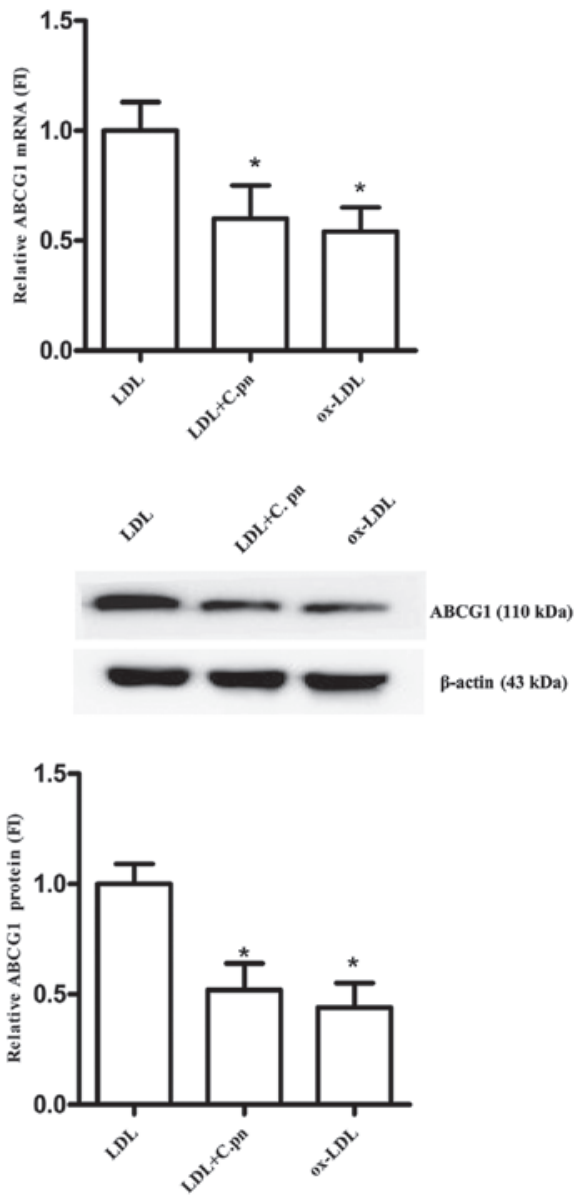

Figure 4. C.pn downregulates ABCA1 and ABCG1 expression in LDL-treated HUVECs. In the presence of 50 mg/1 LDL or ox-LDL, HUVECs were cultured alone or with C.pn (1x106 inclusion forming units) for $48 \mathrm{~h}$. (A and B) Quantitative polymerase chain reaction analysis of (A) ABCA1 and (B) ABCG1 mRNA expression relative to that of $\beta$-actin. (C and D) Western blot analysis of (C) ABCA1 and (D) ABCG1 protein expression relative to that of $\beta$-actin. Data are expressed as the FI of the control (control=1 arbitrary unit). Data are presented as the mean \pm standard deviation from three independent experiments. $\mathrm{P}<0.05$ versus control (LDL group). HUVECs, human umbilical vein endothelial cells; LDL, low-density lipoprotein; ox-LDL, oxidized LDL; C.pn, Chlamydia pneumoniae; ABC, ATP binding cassette transporter; FI, fold induction.

Vascular endothelial cells express receptors for oxidized lipoproteins and exhibit biochemical pathways for sterol synthesis and receptor-mediated endocytosis of lipoproteins (33). A variety of SRs that bind to ox-LDL and promote atherogenesis have been identified. SR-A1 and CD36 promote inflammation and cell adhesion, and lectin-type oxidized LDL receptor 1 (LOX-1) mediates and promotes oxidative stress and inflammation (34). A previous study showed that infection of HUVECs with $C$. pneumoniae enhanced the uptake of ox-LDL and the expression of LOX-1, which may directly contribute to atherogenesis (35). In the present study, C. pneumoniae infection was found to cause a significant increase in SR-A1 and CD36 expression in HUVECs. These findings suggest that these receptors mediate ox-LDL uptake and lead to the accumulation of intracellular TC.

ABCA1 and ABCG1 are considered to be the most important genes involved in reverse cholesterol transport, through the promotion of high-density lipoprotein (HDL) subfraction 3 and/or apolipoprotein A-I-mediated cellular cholesterol efflux (33). Furthermore, HDL is considered to have an important effect on endothelial health (36). A previous study showed that ox-LDL downregulated ABCA1 in human endothelial cells (37). The present study found that $C$. pneumoniae infection downregulated the expression of ABCA1 and ABCG1 in HUVECs, which may have mediated a reduction in cholesterol efflux. Therefore, $C$. pneumoniae infection may impair the efflux of excessive intracellular lipids and lead to the accumulation of intracellular TC.

The accumulation of intracellular TC has cytotoxic effects. Oxidized lipids have been shown to induce oxidative stress-induced endothelial cell apoptosis and reduce mitochondrial function (38). Excess cellular cholesterol is stored as CEs. In the majority of cell types, CEs are present only at low levels and predominantly as cytoplasmic lipid droplets. The appearance of CE-enriched lipid droplets in tissues is a consequence of impaired metabolism or overnutrition (39). ACAT1 is a key and exclusive microsomal enzyme that esterifies FC with fatty acids to form CE. ACAT1 is expressed in numerous tissue and cell types, including hepatocytes and Kupffer cells in the liver, adrenal glands, neurons, macrophages, smooth muscle cells and vascular endothelial cells; this expression accounts for $>80 \%$ of the total ACAT enzyme activity measured in vitro (40). The present study revealed that $C$.pneumoniae increased ACAT1 expression and induced the accumulation of intracellular CE and TC. The increase in intracellular TC induced by $C$. pneumoniae in HUVECs may 
be a result of increased influx and reduced efflux of cholesterol. The increase in intracellular CE may be mediated by ACAT1 and the accumulation of intracellular CE and TC may lead to endothelial dysfunction in atherogenesis.

In conclusion, the present study has revealed that C. pneumoniae significantly induces LDL oxidation and the accumulation of intracellular TC by increasing lipid influx and reducing lipid efflux in HUVECs. In addition, $C$. pneumoniae increases intracellular CE levels. The abnormalities in lipid metabolism induced by $C$. pneumoniae may result in endothelial injury and atherogenesis. These findings may provide an enhanced understanding of the association between infection and atherogenesis.

\section{Acknowledgements}

This study was supported by a grant from the National Natural Science Foundation of China (no. 30900599).

\section{References}

1. Kuvin JT and Kimmelstiel CD: Infectious causes of atherosclerosis. Am Heart J 137: 216-226, 1999.

2. de Boer OJ, van der Wal AC and Becker AE: Atherosclerosis, inflammation and infection. J Pathol 190: 237-243, 2000.

3. Kuo CC, Gown AM, Benditt EP and Grayston JT: Detection of Chlamydia pneumoniae in aortic lesions of atherosclerosis by immunocytochemical stain. Arterioscler Thromb 13: 1501-1504, 1993.

4. Saikku P, Leinonen M, Tenkanen L, et al: Chronic Chlamydia pneumoniae infection as a risk factor for coronary heart disease in the Helsinki Heart Study. Ann Intern Med 116 273-278, 1992.

5. Moazed TC, Kuo C, Grayston JT and Campbell LA: Murine model of Chlamydia pneumoniae infection and atherosclerosis. J Infect Dis 175: 883-890, 1997.

6. Gieffers J, van Zandbergen G, Rupp J, et al: Phagocytes transmit Chlamydia pneumoniae from the lungs to the vasculature. Eur Respir J 23: 506-510, 2004.

7. Summersgill JT, Molestina RE, Miller RD and Ramirez JA: Interactions of Chlamydia pneumoniae with human endothelial cells. J Infect Dis 181 (Suppl 3): S479-S482, 2000.

8. Assmann G and Nofer JR: Atheroprotective effects of high-density lipoproteins. Annu Rev Med 54: 321-341, 2003.

9. Li D and Mehta JL: Oxidized LDL, a critical factor in atherogenesis. Cardiovasc Res 68: 353-354, 2005.

10. Mehta JL: Oxidized or native low-density lipoprotein cholesterol: which is more important in atherogenesis? J Am Coll Cardiol 48: 980-982, 2006

11. Itabe H: Oxidative modification of LDL: its pathological role in atherosclerosis. Clin Rev Allergy Immunol 37: 4-11, 2009.

12. Kalayoglu MV and Byrne GI: Induction of macrophage foam cell formation by Chlamydia pneumoniae. J Infect Dis 177: 725-729, 1998.

13. Kalayoglu MV, Hoerneman B, LaVerda D, Morrison SG, Morrison RP and Byrne GI: Cellular oxidation of low-density lipoprotein by Chlamydia pneumoniae. J Infect Dis 180: 780-790, 1999.

14. He P, Mei C, Cheng B, Liu W, Wang Y and Wan J: Chlamydia pneumoniae induces macrophage-derived foam cell formation by up-regulating acyl-coenzyme A: cholesterol acyltransferase 1. Microbes Infect 11: 157-163, 2009.

15. O'Connell BJ, Denis M and Genest J: Cellular physiology of cholesterol efflux in vascular endothelial cells. Circulation 110 2881-2888, 2004.

16. Collot-Teixeira S, Martin J, McDermott-Roe C, Poston R and McGregor JL: CD36 and macrophages in atherosclerosis. Cardiovasc Res 75: 468-477, 2007.

17. Chang TY, Chang CC and Cheng D: Acyl-coenzyme A: cholesterol acyltransferase. Annu Rev Biochem 66: 613-638, 1997.

18. Caldwell HD, Kromhout J and Schachter J: Purification and partial characterization of the major outer membrane protein of Chlamydia trachomatis. Infect Immun 31: 1161-1176, 1981.
19. Redgrave TG, Roberts DC and West CE: Separation of plasma lipoproteins by density-gradient ultracentrifugation. Anal Biochem 65: 42-49, 1975.

20. Zhu Y, Lin JH, Liao HL, Friedli O Jr, Verna L, Marten NW, Straus DS and Stemerman MB: LDL induces transcription factor activator protein-1 in human endothelial cells. Arterioscler Thromb Vasc Biol 18: 473-480, 1998.

21. Draper HH and Hadley M: Malondialdehyde determination as index of lipid peroxidation. Methods Enzymol 186: 421-431, 1990.

22. Scoccia AE, Molinuevo MS, McCarthy AD and Cortizo AM: A simple method to assess the oxidative susceptibility of low density lipoproteins. BMC Clin Pathol 1: 1, 2001.

23. Gamble W, Vaughan M, Kruth HS and Avigan J: Procedure for determination of free and total cholesterol in micro- or nanogram amounts suitable for studies with cultured cells. J Lipid Res 19: 1068-1070, 1978.

24. Liu W, He P, Cheng B, et al: Chlamydia pneumoniae disturbs cholesterol homeostasis in human THP-1 macrophages via JNK-PPAR $\gamma$ dependent signal transduction pathways. Microbes Infect 12: 1226-1235, 2010.

25. Kunjathoor VV, Febbraio M, Podrez EA, et al: Scavenger receptors class A-I/II and CD36 are the principal receptors responsible for the uptake of modified low density lipoprotein leading to lipid loading in macrophages. J Biol Chem 277: 49982-49988, 2002.

26. Cominacini L, Rigoni A, Pasini AF, Garbin U, Davoli A, Campagnola M, Pastorino AM, Lo Cascio V and Sawamura T: The binding of oxidized low density lipoprotein (ox-LDL) to ox-LDL receptor-1 reduces the intracellular concentration of nitric oxide in endothelial cells through an increased production of superoxide. J Biol Chem 276: 13750-13755, 2001.

27. Roy Chowdhury SK, Sangle GV, Xie X, Stelmack GL, Halayko AJ and Shen GX: Effect of extensively oxidized low-density lipoprotein on mitochondrial function and reactive oxygen species in porcine aortic endothelial cells. Am J Physiol Enolocrinol Metab 298: E89-E98, 2010.

28. Chen H, Li D, Saldeen T and Mehta JL: Transforming growth factor-beta(1) modulates oxidatively modified LDL-induced expression of adhesion molecules: role of LOX-1. Circ Res 89: 1155-1160, 2001.

29. Lin TM, Campbell LA, Rosenfeld ME and Kuo CC: Monocyte-endothelial cell coculture enhances infection of endothelial cells with Chlamydia pneumoniae. J Infect Dis 181: 1096-1100, 2000.

30. Kalayoglu MV, Perkins BN and Byrne GI: Chlamydia pneumoniae-infected monocytes exhibit increased adherence to human aortic endothelial cells. Microbes Infect 3: 963-969, 2001.

31. Vielma SA, Mironova M, Ku JR and Lopes-Virella MF: Oxidized LDL further enhances expression of adhesion molecules in Chlamydophila pneumoniae-infected endothelial cells. J Lipid Res 45: 873-880, 2004.

32. Price DT and Loscalzo J: Cellular adhesion molecules and atherogenesis. Am J Med 107: 85-97, 1999.

33. Hassan HH, Denis M, Krimbou L, Marcil M and Genest J: Cellular cholesterol homeostasis in vascular endothelial cells. Can J Cardiol 22 (Suppl B): 35B-40B, 2006.

34. Apostolov EO, Shah SV, Ray D and Basnakian AG: Scavenger receptors of endothelial cells mediate the uptake and cellular proatherogenic effects of carbamylated LDL. Arterioscler Thromb Vasc Biol 29: 1622-1630, 2009.

35. Yoshida T, Koide N, Mori I, Ito H and Yokochi T: Chlamydia pneumoniae infection enhances lectin-like oxidized low-density lipoprotein receptor (LOX-1) expression on human endothelial cells. FEMS Microbiol Lett 260: 17-22, 2006.

36. O'Connell BJ and Genest J Jr: High-density lipoproteins and endothelial function. Circulation 104: 1978-1983, 2001.

37. Zhu Y, Liao H, Xie X, et al: Oxidized LDL downregulates ATP-binding cassette transporter-1 in human vascular endothelial cells via inhibiting liver $\mathrm{X}$ receptor (LXR). Cardiovasc Res 68: 425-432, 2005.

38. Sata $M$ and Walsh K: Oxidized LDL activates fas-mediated endothelial cell apoptosis. J Clin Invest 102: 1682-1689, 1998

39. Zock PL, Mensink RP, Harryvan J, de Vries JH and Katan MB: Fatty acids in serum cholesteryl esters as quantitative biomarkers of dietary intake in humans. Am J Epidemiol 145: 1114-1122, 1997.

40. Chang TY, Li BL, Chang CC and Urano Y: Acyl-coenzyme A: cholesterol acyltransferases. Am J Physiol Endocrinol Metab 297: E1-E9, 2009. 